\title{
Una prospectiva de la formación en la profesión contable
}

\author{
Hugo Arlés Macías Cardona ${ }^{\mathbf{1}}$
}

\section{Resumen}

El autor hace una recopilación de los principales temas de discusión y reflexión en el campo de la educación contable, tomando como referente las revistas especializadas en el tema que se originan en el mundo académico anglosajón, estableciendo una comparación entre los temas y tendencias en la publicación en Colombia y Estados Unidos e Inglaterra.

El autor concluye que aunque los temas en que se investiga en educación contable no son tan diferentes, es claro que en el mundo anglosajón, la estructura coordinada de trabajo, la disponibilidad de herramientas metodológicas y teóricas y la circulación del conocimiento, son diferentes y configuran un campo de trabajo muy diferente al que se desarrolla en nuestro contexto.

\section{Palabras Clave}

Educación contable, Investigación, Circulación del conocimiento, Docencia.

\footnotetext{
1 Economista de la Universidad Nacional de Colombia y magíster en Economía Internacional de la misma Universidad. Actualmente adelanta estudios doctorales en Administración, Innovación y Desarrollo Territorial. Citar como: Macías, H. A. (2017). Una prospectiva de la formación en la profesión contable. Activos, 15(28), 59-70. DOI: https://doi.org/10.15332/s0124-5805.2017.0028.04
} 


\title{
A prospective of training in the accounting profession
}

\begin{abstract}
The author makes a compilation of main topics of discussion and reflection in the field of accounting education, taking as reference the journals specialized in the subject that originate in the Anglo-Saxon academic world, establishing a comparison between the themes and trends in the publication in Colombia and the United States and England.
\end{abstract}

The author concludes that although the subjects in which accounting education is researched are not so different, it is clear that in the Anglo-Saxon world, the coordinated structure of work, the availability of methodological and theoretical tools and the circulation of knowledge, are different and configure a field of work very different from the one that develops in our context.

\section{Keywords}

Accounting Education, Research, Circulation of Knowledge, Teaching

Buenas noches a todos. Muchas gracias a Jairo Bautista por la invitación a este evento y al equipo editorial de la Revista Activos por organizarlo, a Alejandra Patiño, a Gloria Valero, a Michael Díaz. Es una iniciativa bastante interesante para volverse a encontrar, para volver a intentar construir colectivamente con estos amigos que están hoy en la mesa principal: la profesora Rosa, con quien he tenido la oportunidad de compartir en la Universidad de los Andes; con los estudiantes; con la profesora Ángela, del SENA; con John Cardona, que está en las discusiones contables actuales y estaba allí hace 10 años y hace 20 y hace 30 , es muy muy importante en la contabilidad en Colombia, es en serio, John, todo un personaje para la contaduría pública nuestra, yo llegué hace 15 años y él llevaba muchísimo ya. 
Es importante resaltar que la educación contable es un asunto de construcción colectiva, de construcción nacional, de discusiones que nosotros necesitamos gestar, como se han venido construyendo con distintas alternativas, con distintas visiones desde hace mucho tiempo en Colombia. Nosotros hemos tenido en la contabilidad y en la profesión como contadores públicos muchas viejas discusiones. Me acuerdo en este momento de una frase del profesor John Cardona que citamos en un artículo que escribimos con la profesora Alejandra, que decía en un editorial de mediados de los 80, en el 85 u 86, de la revista de contadores de Antioquia: "Vamos a perder otra década en discusiones y no nos vamos a poner de acuerdo cuando este gremio se mantiene agarrado de todas partes, y nunca nos vamos a poner de acuerdo en avanzar en una construcción colectiva" (Cardona y Zapata, 2006). Y seguimos en discusiones en los noventa, en dos mil, en esta otra década. Yo creo que es el momento de que nos empecemos a encontrar de nuevo.

Cuando, desde la Revista, Gloria Valero y Jairo Bautista nos enviaron las preguntas para el coloquio ( ¿cuáles son las competencias y habilidades que deben desarrollar los estudiantes?, ¿cómo responden los diseños y el desarrollo a los planes de estudio? y ¿cuál es la importancia de la vinculación en los procesos de investigación y proyección social?), decidí centrarme en la última parte, principalmente porque es a lo que me dedico en la academia. Yo trabajo en la Universidad de Medellín desde hace quince años y me mantengo metido en este mundo de las discusiones contables, pero no soy contador. Creo que pocos aquí no son contadores. Mi formación de base es la economía, una maestría en Economía y mi doctorado es en Administración; es una mezcla rara que para lo que más me ha servido ha sido para participar en una comisión del Ministerio de Educación en la que se otorgan los registros calificados, lo cual me permitió comprender las diferentes disciplinas. Justamente trabajo en un programa de Contaduría Pública hace quince años, y cuando entré a ese programa me asignaron la tarea de desarrollar investigación, me dijeron: "Venga, aquí estos contadores no saben investigar, me están diciendo del Ministerio que hagamos una investigación. Usted, que ya es dizque magíster, venga a ver, ayúdenos a 
hacer eso". Entonces llevo quince años intentando entender qué es lo que se hace en investigación contable en Colombia.

Una de las preguntas que se proponía para el coloquio es ¿qué es lo que viene para los contadores ahora? Y una preocupación que tengo aún sin desarrollar es qué se viene para la profesión de contador público en Colombia a nivel internacional y cómo eso tiene que permear los planes de estudio ahora y hacia adelante, porque hay un escenario ya discutido, armado internacionalmente, en el que las cosas ya están claramente identificadas, y otros asuntos donde moverse. Hay un libro que se titula El futuro de las profesiones, que fue publicado en el año 2016 y en el que se demuestra cómo la tecnología cambiará los trabajos calificados: "En una sociedad de Internet no se necesitan médicos, profesores, contadores, arquitectos, sacerdotes, consultores, abogados y muchos otros" (Susskind, 2016).

Como ocurría en el siglo XX, las ovaciones profesionales se convierten en un gran negocio que mantiene profesiones anticuadas opacas, poco accesibles, en las que la experticia de los mejores es disfrutada por unos pocos. Las asociaciones profesionales están siendo reemplazadas por redes de trabajo interdisciplinario mundial (Follet, 1924).

Hay unos reemplazos de funciones tradicionales y ahí está obviamente inmersa la contabilidad, y aquí estoy citando a Parker y Guthrie, que son dos contadores que crearon una de las revistas más importantes de contabilidad, Accounting, Auditing \& Accountability Journal, creada en los años ochenta para editores y lectores. Entonces, en contabilidad hay un reemplazo de las funciones tradicionales por redes autorrealizadas por acreditación distribuida entre pares, por plataformas que interactúan en el conocimiento, por supervisión académica de mercado. Qué viene para el ejercicio profesional del contador público es un asunto que ya está muy estudiado y ya se han identificado asuntos claros que no son fáciles de despachar así en dos o tres frasecitas, sino que hay que sentarse a estudiarlos (Guthrie y Parker, 2016).

Yo quiero hablar especialmente de lo que es la investigación en educación contable. Me quiero centrar un poco en un trabajo que hice 
junto con la profesora Alejandra Patiño, de la Universidad Santo Tomás de Bogotá, y el cual publicamos en la revista Contaduría Universidad de Antioquia (Macías y Patiño, 2014), en el que hicimos un recuento muy sencillo, una descripción de cómo se creó, de cómo evolucionó la revista de Contaduría y Administración, que es la revista con mayor tradición en Colombia; de cómo se creó y cómo ha evolucionado en distintas etapas la revista Cuadernos de Contabilidad, de la Javeriana, que es la revista que hoy formalmente es reconocida como la mejor revista de temas contables en Colombia en términos de revisión de Colciencias; y de cómo se creó y cómo ha evolucionado la Revista Lúmina, de la Universidad de Manizales. Entonces hicimos un montón de cosas sobre esas tres revistas justamente para saber qué ha pasado con esas tres publicaciones, quiénes son los que han escrito, qué vinculación internacional tienen, qué endogamia había, qué temas eran los más tratados, etc.

En ese trabajo, que fue publicado en la revista Contaduría Universidad de Antioquia, encontramos con la profesora Patiño que educación contable es el tema sobre el que más se investiga en Colombia y sobre el que más se publica en esas tres revistas (que son las principales revistas contables de Colombia) (Macías y Patiño, 2014).

Sin embargo, es importante resaltar que hay otro grupo de revistas, y muy cerca de ellas está la Revista Activos, que es la que está organizando este evento, cuyas publicaciones versan principalmente sobre el tema de educación contable, entonces, no es un tema menor, es un tema al que se dedica mucha gente, por ejemplo, el profesor John Cardona, desde hace mucho tiempo, con los profesores de aquí, con unos profesores de la Nacional desde hace menos, pero de manera muy juiciosa.

Por ejemplo, a mí me gustan mucho los trabajos de la profesora Fabiola Loaiza, que está con nosotros en el auditorio, y que son unos trabajos muy juiciosos, muy rigurosos. Y el elemento que más me gusta del trabajo que hace la profesora Fabiola es que tiene en cuenta lo que dicen los otros sobre educación contable en Colombia, que es un problema que la academia contable tiene (Loaiza, 2011 y 2013). 
En contabilidad, en la academia contable colombiana, cada uno escribe como si nunca nadie hubiera hablado de eso. Entonces alguien escribe de educación contable y otro escribe sobre educación contable y otro escribe de educación contable... y todos los trabajos están desconectados. El trabajo de la profesora Fabiola Loaiza es muy valioso en ese sentido. Y hay un trabajo de un profesor muy joven que aún desconocemos, que tiene el mismo rigor del trabajo de la profesora Fabiola, es un profesor de Armenia que se llama Marlon García Jiménez. Marlon también tiene un trabajo muy bonito, integrador, acerca de que se está haciendo en la educación contable en Colombia, y hace un trabajo empírico muy riguroso, muy juicioso, distinto a los otros trabajos (García, 2017). Entonces, son como unas nuevas miradas integradoras, las de la profesora Fabiola y el profesor Marlon. Marlon todavía es muy desconocido, los invito a mirarlo, a leerlo y a seguirlo; el profesor Marlon trabaja en la Universidad La Gran Colombia de Armenia; formado en la Universidad del Quindío, es una persona interesada en el tema de la educación contable.

Ellos tienen la mirada nacional desde la mirada crítica de la profesora Fabiola. Por un asunto de tradición en la academia contable colombiana, por la Federación Nacional de Estudiantes de Contaduría Pública de Colombia [FENECOP] y por toda una tradición política, la academia contable colombiana ha estado de espaldas a la academia internacional, y principalmente a las vertientes anglosajonas. Los principales referentes internacionales de la academia contable en Colombia no sobrepasan los límites de la América Latina; en ese marco nos movemos muy cómodamente, y todo el mundo va hasta México, a Chile, a Argentina, y en algunos casos se refieren a autores españoles que han tenido una fuerte influencia en la academia local. Es claro que la principal limitante es idiomática, y solo en los últimos años se han explorado trabajos y avances generados en universos diferentes al regional.

Pero cuando uno no va a lo anglosajón, ahí sí la cosa se complica un poco; ya la profesora Rosa está yendo a los encuentros de la Academia Americana de Contabilidad, ojalá nosotros fuéramos a eso, ojalá fuéramos a los encuentros anuales de la Asociación Europea de Contabilidad, como lo 
hacen decenas de académicos de México, decenas de académicos de Brasil; pero ahora no estamos yendo y no estamos participando, cuando hemos tenido a esa gente aquí.

El presidente de la Academia Americana de Contadores, Estefen Zeff, ha venido a Colombia, ha publicado varias veces en la revista de contadores de allá de Antioquia y mantiene un contacto permanente con el profesor John Cardona, como mantiene contacto con otros académicos distintos de América Latina. Entonces, mi punto es este: como respuesta a esta tradición colombiana que lleva 30 años y que es muy respetable y muy defendible, nosotros hemos estado de espaldas a la academia anglosajona. Yo lo que quiero dejar aquí es una inquietud sobre pensar la educación contable en esa academia colombiana, pero con una conexión hacia la anglosajona, mirar cómo es la investigación contable en las revistas anglosajonas, cuáles son los temas que se trabajan en educación contable en el mundo anglosajón.

Pero no es como describen muchos de los colegas, que dicen "miren, les traigo una idea, se me ocurrió esta mañana mientras me estaba bañando". No, eso ya se ha trabajado. Uno de los trabajos que ya se han hecho sobre eso y que se centra en la conexión de la educación contable con el mundo anglosajón lo hicieron la profesora Alejandra Patiño y la profesora Gloria Valero y lo publicaron en un capítulo de libro en 2010 (Patiño y Valero, 2010). Los capítulos de libro tienen la restricción de que circulan muy poco. Cuando me enteré de que existía, de ese capítulo de libro, tuve que pedirles a ellas que me mandaran el PDF, porque, díganme, yo a dónde consigo ese capítulo del libro, que es muy complicado de conseguir. Entonces está ese capítulo de libro en el que se hace una conexión; es decir, lo que ellas se preguntaban era sobre los aspectos de la educación contable que son objeto de estudio en los journals.

Otra persona que ha hecho un esfuerzo por mirar la educación contable y las conexiones con lo anglosajón es un profesor formado en la Universidad Nacional de aquí, de Bogotá, y es el profesor Freddy León Paime, que trabajó mucho tiempo en la Universidad Militar y que ahora está en la Tadeo. Freddy 
también tiene un par de trabajos en los que conecta la educación contable nuestra con el mundo anglosajón desde otra perspectiva.

Yo quiero focalizar el asunto, pero primero voy a mencionar esto: el trabajo de las profesoras Alejandra Patiño y Gloria Valero tiene este título: "Aproximación a la educación contable a través de una revisión de journals internacionales". Es un capítulo del libro de Yesid Pérez Meneses e Irma Ramos Moreno titulado El rol de la teoría y la investigación contable en el desarrollo de la sociedad (Patiño y Valero, 2010). Lo que hicieron las autoras fue una revisión bibliográfica para saber cómo se mueve eso en los journals, en bases de datos como Ebsco, Emerald y en ScienceDirect, y ellas identificaron 57 artículos en 23 revistas internacionales, entre las que se destacan dos revistas que están especializadas en educación contable y que no son las únicas en inglés que tratan este tema; estas revistas ya tienen unas discusiones enormemente avanzadas.

Y nosotros, en la academia contable colombiana, en general, estamos desconectados de eso, que es un ejercicio que está muy avanzado. Entonces, las profesoras Alejandra Patiño y Gloria Valero llegaron aquí, identificaron estas, que son unas revistas que también tiene identificadas el profesor Freddy León Paime. Las profesoras Gloria y Alejandra, en ese capítulo del libro, llegan a decir "mire, estos son los aspectos identificados en las revistas en inglés de contabilidad, y en cuanto al tema de educación contable, de lo que se habla es de esto, del plan de estudio, de desempeño y prospectiva profesional, de metodologías de enseñanza, de docencia universitaria, de productividad académica contable, de ética, de historia contable, aspectos generales sobre educación". Como ustedes ven, ellas hablan de un tipo de revistas, de las cuales solamente esas dos están especializadas en educación, las otras son revistas de contabilidad o de áreas cercanas, pero no de educación, aunque ellas buscaron artículos de educación contable. A lo que yo quiero ir es a que hay unas revistas que están especializadas en educación contable, y la indicación que yo quiero hacer a los académicos de educación contable, que son la mayoría en Colombia, es que leamos esas 
revistas, que miremos cuáles son las discusiones y que miremos un poco esa misma pregunta que se hacían las profesoras Gloria y Alejandra, que es buena: ¿sobre qué se habla en educación contable?

Las principales revistas de educación contable son:

- Journal of Accounting Education

- Accounting Education

- Issues in Accounting Education

- Advances in Accounting Education

- Accounting Education and Journal of Theory Practice and Research

- Accounting Education: An International Journal

- Global Perspectives on Accounting Education

- The Accounting Educators' Journal.

A lo que voy, mis amigos, es a que estas revistas están especializadas en educación contable: todos los artículos que salen ahí son todos de educación contable. Los editores de estas revistas, que son casi todas de los Estados Unidos, trabajan en universidades distintas en áreas diferentes de los Estados Unidos; cada dos años hacían un balance de lo que estaba pasando con la educación contable, recientemente el balance se hace de manera anual. Los editores de estas revistas hacen un artículo conjunto - ahora lo hacen cada año- sobre qué se ha dicho en el último año en educación contable en estas revistas de educación contable; ellos hacen un balance, hacen una revisión en la que usted puede ver cuáles son las tendencias en cada uno de los subcampos de la educación contable, y, como vamos a ver, tienen mucho en común con respecto a lo que se hace aquí. 
Lo que nosotros hacemos aquí, desde mi punto de vista, está muy desestructurado, muy suelto. Por eso me gustan mucho los trabajos de Marlon y de la profesora Fabiola, porque intentan integrar lo que se hace en la academia contable nacional. Los trabajos de la profesora Alejandra y los otros amigos de aquí de Bogotá están concentrados en una parte, que es el currículo, en una parte de la educación contable, aunque son muy profundos. En eso hay una parte que ellos no trabajan, que es también otra manera de hacerlo.

De manera que sabemos cuáles son los temas y las líneas de investigación que se manejan en esos journals, porque se han hecho revisiones. Y en esas publicaciones se pueden encontrar cada vez más artículos que abordan el tema de la evaluación. Entonces, es claro que la evaluación es un tema tratado en esas revistas desde hace varias décadas y es una de las categorías centrales en investigación en educación contable en esos journals.

Otro tema lo componen los planes de estudio y los métodos de enseñanza, que hasta hace algunos años se llamaban planes de estudio, aseguramientos de aprendizaje y métodos de enseñanza. Al respecto, ustedes ven los cambios. Hay un tema que siempre se ha llamado igual: es tecnología educativa, que no se trabaja en Colombia. Otra sublínea la conforman los profesores, los asuntos profesorales, y al respecto hay todos unos métodos, todas unas teorías, todos unos temas para trabajar, y de manera estructurada con discusiones, no solo las que se han dado recientemente, sino que han venido dándose hace mucho tiempo y se han consensuado por medio de los editores de estas revistas.

Otros temas son estudiantes, plan de estudios y métodos de enseñanza. Los estudiantes constituyen otro tema, los planes de estudios conforman otro tema y lo mismo ocurre con los métodos de enseñanza. Entonces, lo que yo quiero es invitarlos, investigadores de educación contable, a que miremos si este mapa en el que los editores de las revistas han incluido las investigaciones de educación contable crean un escenario de convergencia entre los diferentes investigadores, crea temas comunes de diálogo y 
conversación. El reto está en cómo nosotros podemos proponer artículos para publicar en estas revistas de perfil internacional.

En síntesis, lo que les quería señalar, mis amigos, es que busquemos la manera de integrar lo que hacemos con las grandes discusiones que hay en educación contable. Celebro bastante que la Revista Activos quiera especializarse en educación contable, que es uno de los problemas que tenemos en las revistas contables colombianas, porque en todas se publica de todo y entonces ninguna es citada, porque ninguna se vuelve referente en nada, sino que todas se lanzan a publicar en una amplia variedad de temas. Es muy importante que una revista diga "vamos a publicar sobre educación contable, vamos a mirar si esto nos sirve". Puede ser que después de hacer un análisis digamos "no" o “¿cómo así?, eso es por allá, nosotros vamos a hacer otra cosa".

\section{Referencias}

Cardona, J. y Zapata, M. A. (2006). Educación contable: antecedentes, actualidady prospectiva. Medellín: Universidad de Antioquia.

Follet, M. P. (1924). Creative experience. London: Longmans, Green and Co.

García, M. (2017). Antecedentes nacionales e internacionales sobre didáctica específica de la disciplina contable en el siglo XXI. Contexto, 6, 122- 134.

Guthrie, J.y Parker, L. (2016). Whither the accounting profession, accountants and accounting researchers? Commentary and projections. Accounting, Auditing \& Accountability Journal, 29(1), 2-10.

Loaiza, F. (2011). Producción académica sobre educación contable en Colombia 2000-2009: incidencia de la pedagogía crítica. Lumina, 12, 172- 194.

Loaiza, F. (2013). Corrientes educativas internacionales presentes en programas de contaduría pública. Cuadernos de Contabilidad, 14, 189- 215.

Macías, H. A y Patiño, R. A. (2014). Evolución de las revistas contables colombianas: de la reflexión a la investigación. Contaduría Universidad de Antioquia, 64, 13-48.

Patiño, A. y Valero, G. (2010). Aproximación a la educación contable a través de la revisión de journals internacionales. En Y. Pérez Meneses e I. Ramos, El rol de 
la teoría y la investigación contable en el desarrollo de la sociedad (pp. 1-24). Bogotá: Editorial Universidad Cooperativa de Colombia.

Pérez, Y. y Ramos, I. (2010). El rol de la teoría y la investigación contable en el desarrollo de la sociedad. Bogotá: Editorial Universidad Cooperativa de Colombia.

Susskind, R. (2016). El futuro de las profesiones: cómo la tecnología transformará el trabajo de los expertos humanos. España: Teell Editorial. 